Théologiques

Théologiques

\title{
L’altermondialisation et la théologie au Brésil
}

\section{Agenor Brighenti}

Volume 18, numéro 1, 2010

Les Églises chrétiennes et la mouvance altermondialiste

URI : https://id.erudit.org/iderudit/1003546ar

DOI : https://doi.org/10.7202/1003546ar

Aller au sommaire du numéro

\section{Éditeur(s)}

Faculté de théologie et de sciences des religions, Université de Montréal

\section{ISSN}

1188-7109 (imprimé)

1492-1413 (numérique)

Découvrir la revue

\section{Citer cet article}

Brighenti, A. (2010). L’altermondialisation et la théologie au Brésil.

Théologiques, 18(1), 65-92. https://doi.org/10.7202/1003546ar

\section{Résumé de l'article}

Afin d'aborder l'altermondialisation et la théologie au Brésil, l'auteur concentre sa réflexion sur trois points. En premier lieu, « Théologie et Église au Brésil » apporte quelques éléments de l'itinéraire d'une Église libératrice dont la place a changé au sein de la société, ce qui, en conséquence, a changé le lieu et la fonction propre de la théologie elle-même. En second lieu, « Théologie et altermondialisation » explicite les bases d'une théologie brésilienne qui a contribué à créer un pont entre la foi chrétienne et la société, selon un principe d' « insertion par contraste » en celle-ci, afin de faire de la foi chrétienne et de sa théologie elle-même une prophétie. Enfin, dans " Altermondialisation et théologie ", l'auteur présente, dans leurs grandes lignes, les éléments qui caractérisent l'altermondialisation dans la réflexion théologique au Brésil. 


\title{
L'altermondialisation et la théologie au Brésil*
}

\author{
Agenor BRIGHENTI" * \\ Théologie \\ Université catholique de Curitiba, Brésil
}

Un «autre monde » n'est pas seulement possible, il est urgent et nécessaire. Possible, car, d'un côté, «l'imaginaire appartient également à la réalité, à sa meilleure partie » (Boff 1996, 7, nous traduisons) et, de l'autre, le système libéral capitaliste n'est pas la «fin de l'histoire », contrairement à ce qu'a postulé Fukuyama (1992). Quel sera ce monde, nul ne le sait. La seule certitude est que ce monde se doit d' «être autre» (de Sousa Santos 2009a et b). Un monde où tous y trouvent leur place, dans le souci et la protection de la biodiversité de la planète, notre «maison» commune. Autrement dit, un monde différent du monde actuel, qui est fait d'«îlots de prospérité dans un océan de pauvreté » (Salgado 2001, nous traduisons).

L'Église du Brésil, ainsi que d'autres Églises, ont professé, depuis plus de quatre décennies, un espoir actif. Elle a espéré, contre tout espoir, que soit recherché et édifié "un autre monde», sans exclusions, juste et solidaire. Le chemin n'a pas été facile. Outre les difficultés inhérentes à la cause, comme la crise des utopies, le manque de solutions de rechange par rapport au système libéral capitaliste et la fragmentation croissante du tissu

* Texte traduit du brésilien par la rédaction.

** Agenor Brighenti est professeur à l'Université catholique de Curitiba (PUCPR), Brésil. Il est également président de l'Institut national de pastorale de la Conférence nationale des évêques du Brésil, professeur invité à l'Université pontificale du Mexique (UPM) et à l'Institut théologique-pastoral d'Amérique latine du Conseil épiscopal latino-américain (CELAM). Ses recherches récentes s'orientent vers les implications de la crise de la modernité et de la culture urbaine pour le travail de l'Église dans la société excluante actuelle et ses répercutions sur la théologie latino-américaine. Ses dernières publications incluent (2006) A pastoral dá o que pensar. A inteligência da prática transformadora da fé, São Paulo (Brésil)/Mexico, Paulinas/Dabar; (2010), dir., A missão em debate. Provocações à luz de Aparecida, São Paulo (Brésil)/San Pablo (Colombie), Paulinas. 
social, les obstacles internes ou externes à l'Église n'ont pas manqué. À l'extérieur, d'une part, il y a eu la répression qu'orchestrèrent certaines dictatures militaires dans les années 1960 et 1970, avec, comme conséquence, le martyre de nombreuses personnes. Aujourd'hui cette répression a été remplacée par un marché et un commerce international dépourvus d'éthique, qui privilégient le profit et le système financier. À l'intérieur, nombreux furent les chrétiens engagés parmi des milieux populaires en quête d'un "autre monde possible ", mais qui furent incompris. Parmi eux, des théologiens qui furent soupçonnés ou réduits au silence, et des évêques qui furent victimes de diffamation ou d'autres injustices.

Cependant, rien de tout cela n'a empêché une vigoureuse pastorale sociale, cherchant à faire des pauvres les sujets d'une société nouvelle, juste et solidaire, ainsi qu'une réflexion théologique créative et un travail systématique de formation de la citoyenneté qui est en train de contribuer à l'articulation d'une société civile mondiale, en vue d'un «autre monde possible».

Dans la présentation qui suit sur l'altermondialisation et la théologie au Brésil, nous nous limiterons à trois points. En premier lieu, "Théologie et Église au Brésil » apportera quelques éléments relatifs à l'itinéraire d'une Église libératrice qui a vu sa place changer au sein de la société et, conséquemment, le lieu et la fonction propre de la théologie elle-même. En deuxième lieu, "Théologie et altermondialisation» explicitera les bases d'une théologie brésilienne qui a contribué à créer un pont entre la foi chrétienne et la société, selon un principe d' «insertion par contraste» en celleci, afin de faire de la foi chrétienne et de sa théologie elle-même une prophétie. Enfin, dans "Altermondialisation et théologie », nous présenterons, dans leurs grandes lignes, les éléments qui caractérisent l'altermondialisation dans la réflexion théologique au Brésil.

\section{Théologie et Église au Brésil}

Avant d'aborder le sujet de l'altermondialisation dans la théologie produite au Brésil, il faut d'abord noter que les théologiens, axés sur la perspective de la libération, admettent que leur théologie n'est pas un simple produit de la spéculation professionnelle - opinion partagée par des voix d'autres confessions. Celle-ci est, avant tout, une réponse aux questions posées par les personnes, chrétiennes ou non, engagées dans une situation sociohistorico-pastorale qui contredit le message évangélique. Cela signifie un changement de lieu et de fonction pour la théologie, fruit du changement de la place de l’Église dans la société. 


\subsection{Le nouveau lieu de la théologie}

Quel est le lien entre la théologie et l'altermondialisation? Comment faire le rapprochement entre la réflexion sur la foi et l'engagement historique des chrétiens dans l'édification d'un "autre monde possible" ? Pour Leonardo et Clodovis Boff (1986, 24-25), la théologie peut être comparée à un arbre dont les branches correspondent aux théologiens professionnels, reliés au tronc qui représente la réflexion des pasteurs et des agents de la pastorale, eux-mêmes soutenus et nourris par les racines de l'arbre qui représenteraient l'action et la réflexion — vitale et concrète, bien que «souterraine» et anonyme - de milliers de communautés qui vivent leur foi dans une perspective libératrice. Il s'agit d'une théorie de l'action des pratiques ecclésiales, une théorie incarnée, pénétrée de sa spiritualité, de sa liturgie et de son éthique. De la base jusqu'au niveau le plus élevé, il existe un même flux continu de pensée, un même processus théologal global. Chacun de ces niveaux ou lieux reflète la même expérience: la foi confrontée à l'injustice et à l'exclusion. Ce qui réunit les trois niveaux est la même foi transformatrice de l'histoire, autrement dit la compréhension de l'histoire dans l'optique d'une foi transformatrice. La distinction entre ces niveaux se situe uniquement au plan logique et, plus concrètement, dans le langage. Ce sont les trois « $\mathrm{P}$ » de la théologie latino-américaine: la théologie populaire, la théologie pastorale et la théologie professionnelle (Boff 1990, 91-93), trois types de réflexions théologiques, non isolés et non juxtaposés. Ils sont intégrés entre eux et chacun contribue à l'intégration des autres niveaux ou lieux. La tâche du théologien n'est autre que l'art d'articuler le discours de la société et du monde des significations populaires avec le discours de la foi et de la grande tradition de l'Église.

Ainsi, si l'intelligence de la foi s'articule à partir de l'expérience communautaire de cette même foi, le véritable lieu de la théologie n'est pas le monde académique, mais la communauté ecclésiale, qui s'insère "par contraste » au sein d'une société injuste et excluante. D'ailleurs, on retrouve ici une des particularités de la théologie latino-américaine, incompréhensible en dehors de ces circonstances. Avant toute élaboration plus sophistiquée, celle-ci naît de la nécessité vitale de penser de façon théologique l'expérience vivante et concrète de la communauté ecclésiale. Il y a, en premier lieu, l'expérience communautaire de la foi, puis il y a la théologie et son intelligence réfléchie — «moment second », il est vrai, mais non secondaire, car indispensable comme effort de réflexion pour éclairer l'expérience complexe de la foi dans un contexte concret. 
L'évolution postérieure et, par-dessus tout, l'ensemble des obstacles auxquels la théologie latino-américaine a été confrontée, laisseraient dans la pénombre ce donné simple - à première vue sans grande importance - : la vie de la communauté ecclésiale en tant que «lieu naturel» de la théologie. De plus, la théologie s'affirme, non seulement comme une réalité inséparable de la conscience vivante de l'Église, mais également comme la conviction réfléchie que la vie et l'expérience d'une communauté ecclésiale concrète précèdent la théologie (Palácio 2000, 51-64).

Pour cela, une théologie authentique sera toujours un «moment second ", le moment théorique de la vie et de l'agir ecclésiaux. La théologie latino-américaine est une théologie originale dans son contexte, non nécessairement par sa méthode et encore moins par son produit final, mais avant tout par l'expérience ecclésiale qui la soutient. L'essentiel de ce paradigme théologique n'est pas la théologie mais la libération, l'expérience incarnée de la foi, dans une perspective transformatrice. C'est à partir de ce point que naît la théologie comme intelligence de la foi, de manière délibérée, intentionnelle et réfléchie dans, à partir de, et pour le contexte de cette même expérience de la foi. Sa particularité ne se trouve pas dans la théologie en tant que telle, ni dans sa sémantique et encore moins dans sa syntaxe, mais avant tout dans l'expérience ecclésiale de laquelle elle vit et pour laquelle elle recherche des voies qui répondent aux défis concrets. Enfin, notre théologie est innovatrice par rapport aux autres, à cause de sa fonction et de son lieu particuliers, car elle ne s'articule pas à partir de l'Académie, de questions théoriques ou de questions creuses débattues entre théologiens. Les bonnes idées, pas plus en théologie qu'ailleurs, ne tombent pas du ciel: elles jaillissent de la réalité. C'est donc l'expérience de la foi des communautés ecclésiales qui donne à penser à la théologie.

\subsection{La nouvelle place de l'Église}

Comme il est possible de le constater, si la théologie latino-américaine est l'intelligence réfléchie des pratiques libératrices des chrétiens au sein de la société, son nouveau lieu et sa nouvelle fonction sont dus à la place que l'Église en est venue à occuper dans la société.

En Amérique latine, incluant le Brésil, une réflexion théologique engagée dans la recherche et dans la réalisation d'un «autre monde possible» résulte de la complicité de plusieurs facteurs sociaux et ecclésiaux, sans lesquels ceci n'aurait pu être possible. 
Dans le milieu social, le «mythe du progrès » et l'utopisme généré dans l'Europe de l'après-guerre, a créé en Amérique latine, lors de son récent processus d'industrialisation - plus particulièrement dans les milieux intellectuels et dans les couches populaires - un climat de changement et d'attente de conquêtes sociales. Dans un premier temps, le populisme (de Getúlio Vargas au Brésil et de J. D. Perón en Argentine) a canalisé ces aspirations dans un programme «développementiste» national. Étant donné que l'avancée du capitalisme sauvage a montré que ceci était illusoire, alors la théorie de la dépendance, élaborée par des théoriciens de la Commission économique pour l'Amérique latine de l'ONU (CEPAL), s'est opposée à la "théorie du développement", en montrant que le développement du Sud impliquait une dépendance accrue vis-à-vis du Nord. L'unique solution était de rompre avec la dépendance. Des coups d'État militaires ont toutefois suivi, largement soutenus par la CIA. Néanmoins, à mesure qu'augmentait la répression, augmentait aussi la conscience libératrice. Au Brésil, les ligues paysannes (Francisco Julião), la lutte syndicaliste (Lula), l'éducation populaire (Paulo Freire), la mobilisation des jeunes étudiants (Juventude Universitária Católica - JUC), etc., se sont chargés de donner une organisation à la résistance.

Dans le milieu ecclésial, l'ouverture de l'Église à la modernité avait créé un nouveau climat, particulièrement avec les encycliques du pape Jean XXIII, Mater et Magistra, ainsi que Pacem in Terris, aboutissant à la convocation du concile œcuménique Vatican II. L'idéal de Jean XXIII, qui était d'en arriver à une Église des pauvres, afin qu'elle soit une «Église de tous», a trouvé, en Lumen Gentium et Gaudium et Spes, les fondements d'un aggiornamento ecclésial déjà tardif. Par la suite, Paul VI, avec Populorum Progressio (1967) — qui montre le sous-développement du Sud comme sous-produit du développement du Nord - , aura une influence directe sur Medellín (1968), qui fera une réception créative de Vatican II. Sur le continent latino-américain, l'Action catholique spécialisée, particulièrement la Jeunesse universitaire catholique (JUC), ainsi que la Jeunesse ouvrière catholique (Juventude Operária Católica, JOC), fondées sur la "nouvelle théologie » de Chenu, Lubac et Congar et sur les idées sociales de Mounier et Lebret, ont aidé les chrétiens à passer de l'engagement chrétien dans le social en tant que "conversion des consciences" à une "conversion des structures sociales». 
C'est dans ce contexte qu'est née une Église libératrice, qui a trouvé dans les Communautés ecclésiales de base (CEB) son expression la plus authentique. Ce sont elles qui ont peu à peu fait jaillir :

1) la théologie de la libération, centrée sur l'option pour les pauvres;

2) la pastorale sociale, avec ses multiples services et, tout particulièrement dans le secteur rural, dans celui des droits humains et auprès des indigènes;

3) l'insertion des religieux dans les banlieues urbaines, particulièrement les plus défavorisées;

4) la lecture populaire de la Bible, etc.

Elles ont également eu une grande influence sur la nouvelle place de l'Église dans la société, à travers des mouvements comme "Chrétiens pour le Socialisme» (Cristaos para o Socialismo) et le Vicaria de la Solidaridad au Chili, «Prêtres pour le Tiers Monde»(Sacerdotes para o Terceiro Mundo) en Argentine, et la Jeunesse universitaire catholique au Brésil. Ces pratiques, d'ordre pratique ou théorique, ont créé une nouvelle façon d'être Église, fondée sur la compréhension de l'articulation de la foi avec son contexte humano-historique de construction de la justice, soulignant la dimension sociale et politique de la foi et de l'évangélisation, tout comme la responsabilité éthique des chrétiens dans la transformation du cadre inique d'injustice structurelle.

Un fait important est que cette posture rencontrera une articulation organique avec des conférences nationales ou continentales des religieux (CLAR) et dans des pays comme le Brésil et le Chili, avec des conférences nationales d'évêques (Conferência Nacional dos Bispos). Dans plusieurs lieux, cette nouvelle forme d'être Église a créé des polarisations internes, particulièrement en Argentine puis au Chili, aggravant la situation de ces chrétiens engagés qui, bien que réprimés par les régimes autoritaires d'exception, ont également été persécutés par l'Église même qu'ils ont servie à travers un travail dur et risqué avec les pauvres.

Le prophétisme de l'Église au Brésil est surtout dû à l'engagement social des chrétiens dans des services pastoraux, tels que: la pastorale ouvrière, qui a contribué à la reprise du syndicalisme; la pastorale de la terre, qui a joué le rôle principal dans la lutte pour la réforme agraire, l'agro-écologie et l'agriculture familiale, favorisant la création du Mouvement des sans-terre (Movimento dos Sem-Terra); le Conseil indigéniste missionnaire (CIMI), organisme de la Conférence des Évêques qui a pris la défense des indigènes contre l'expansion des grandes propriétés; la Commission Justice et Paix 
qui a joué un rôle prépondérant dans la lutte pour les droits humains; les Semaines sociales qui ont contribué à la formation d'un laïcat inséré dans le monde; etc. Les CEB fournissent l'appui mystique et organisationnel, soutenues par une lecture populaire de la Bible dans une perspective ocuménique, que promeuvent des initiatives telles que celles de Carlos Mesters et de Milton Schwantz.

Cette Église, qui assume une nouvelle place dans la société et qui joue le rôle prophétique, n'aurait jamais pu exister sans l'audace et la lucidité de grands évêques comme Dom Hélder Câmara (Recife), Dom Paulo Evaristo Arns (São Paulo), Dom Alö̈sio Lorscheider (Fortaleza), Dom Ivo Lorscheiter (Santa Maria), Dom José Gomes (Chapecó), Dom Pedro Casaldáliga (São Felix), Dom Tomás Balduíno (Goiás Velho), Dom Fragoso (Crateús), Dom Waldir Calheiros (Volta Redonda), Dom Moacir Grechi (Rio Branco), Dom José Maria Pires (João Pessoa), Dom Luciano Mendes de Almeida (Mariana), etc. Malheureusement, les nominations, dans les trente dernières années, hormis quelques rares exceptions, ont démantelé la Conférence nationale des Évêques du Brésil et renforcé un processus de restauration ecclésiale, dans une perspective pré-conciliaire. À l'arrièregarde de ces évêques et des vastes segments de communautés ecclésiales insérées prophétiquement au sein d'une société injuste et excluante, il y eut des théologiens de l'envergure de L. Boff, J. Comblin, H. Assmann, C. Boff, J. B. Libânio, A. Antoniazzi, O. Beozzo, L. C. Susin, B. Ferraro, etc.

Paradoxalement, les années 1990 ont été marquantes, au même titre que la période des transformations et de crises des années 1960, avec un facteur d'autant plus aggravant, tel que le fut la crise holistique - tant dans le premier monde que dans le tiers monde: crises de civilisations, d'utopies, d'idéologies, des métarécits, des paradigmes, du sens (éthique), etc. C'est la crise de ce que l'on appelle «la raison technico-instrumentale ", qui perdure de nos jours, avec de sérieuses conséquences, tant pour l'expérience religieuse que pour la réflexion théologique. La chute du mur de Berlin était le référentiel symbolique des grands changements de ces années. Plus tard, les attentats du 11 septembre et le Forum social mondial sont les référentiels symboliques de cette nouvelle période, mettant en évidence l'épuisement du modèle libéral capitaliste et l'irruption d'une société civile mondiale occupant le vide de l'éclipse des sujets sociaux. À la base, il y a deux phénomènes aux proportions gigantesques: l'émergence d'une conscience planétaire et la découverte des cultures. Le premier fait volatiliser les particularismes et nationalismes en imposant une nouvelle conceptualisation, tant de la particularité que de l'universalité. La mondialisation 
aujourd'hui, outre le domaine économique, qui possède dans la globalisation son côté pervers par le moyen du marché total, passe également par la techno-science, surtout par la robotique et l'informatique, par la stratégie militaire, par la spiritualité et par la politique. Le deuxième rompt avec le mythe de la culture supérieure, disqualifiant toute tentative d'ethnocentrisme. Dans la perspective de Levinas, la découverte des cultures est également la prise de conscience de l'irruption de l'autre en tant que gratuité, et de la dimension sabbatique de l'existence. L'autre, avant d'être un impératif éthique, tel que la morale moderne kantienne l'avait diagnostiqué, est un horizon de réalisation et, en conséquence, d'auto-identification, d'autocompréhension et de sens. Nous sommes avec les autres. Dans un tel contexte, la religion devient l' 'âme» des cultures, plaidant, au-delà du dialogue interculturel, pour le dialogue interreligieux. Il s'agit de deux phénomènes qui font surgir de nouvelles questions, pour lesquelles nous ne pouvons plus continuer de donner les mêmes réponses.

\section{Théologie et altermondialisation}

Répétons notre question: quel est le lien entre la théologie et l'altermondialisation? Comment faire le rapprochement entre la réflexion sur la foi et l'engagement historique des chrétiens dans l'édification d'un «autre monde possible» ? Comme nous l'avons vu, la relation théologie-altermondialisation au Brésil est due au nouveau lieu et à la nouvelle fonction de l'intelligence de la foi, elle-même déterminée par la nouvelle place que l'Église a occupée dans la société, en tant que promotrice - de par l'engagement citoyen des chrétiens — d' « un autre monde possible». Bien entendu, la fonction de la théologie ne se résume pas aux frontières de l'action ecclésiale. En partant du fait que l'Église n'existe pas pour elle-même, mais qu'elle appartient au monde et existe pour le salut de ce dernier, tant les pratiques ecclésiales que la théologie doivent se rencontrer au sein de la société. L'interaction des deux amène à situer le peuple de Dieu comme pèlerin au sein d'une même et unique humanité pérégrinante. La nouvelle place de l’Église dans la société et donc le nouveau lieu et la nouvelle fonction de l'intelligence de la foi font de la foi et de la théologie elle-même une prophétie.

\subsection{L'action ecclésiale comme prophétie}

Théologie et altermondialisation ont à voir avec action ecclésiale et prophétisme. Lors de son « retour aux sources ", le concile Vatican II a lui- 
même compris l'Église et sa mission dans l'indissociabilité du trinôme Église-Royaume-monde. Il n'y pas d'Église sans Royaume de Dieu et sa mission est d'être sacrement de ce Royaume dans le monde, ce qui la décentre d'elle-même (Sobrino 2006, 283-288). Le Royaume de Dieu n'existe pas uniquement dans l'Église, en tant que communauté des rachetés, socialement construite. De même qu'il ne se réalise pas uniquement dans l'intériorité secrète de la conscience, dans la subjectivité religieuse méta-historique, mais il se produit dans le concret de la réalisation de l'amour du prochain, au-delà de l'ambiguïté de l'histoire, en ses objectivations empiriquement perceptibles. En conséquence, la mission de l'Église, celle de rendre possible le Royaume de Dieu, se réalise dans le monde et pour le monde.

En outre, l'interaction Église-Royaume-monde se situe dans une relation de tension, marquée par une distance de l'Église face au monde et, en même temps, d'insertion dans celui-ci. C'est uniquement lorsque l'Église s'éloigne d'un monde injuste et marqué par l'exclusion et, en même temps, qu'elle y adhère dans une perspective libératrice, qu'elle devient sacrement de salut du monde (Bigo et Ávila 1986, 111-119). Il s'agit là d'une insertion «par contraste». Une action pastorale d'incarnation, dans la mesure où elle assume pour racheter, est toujours signe de contradiction face à n'importe quelle situation d'injustice et d'exclusion (Muñoz 2006, 345-352).

En réalité, la prophétie de l'action ecclésiale se réalise dans le mode d'insertion de l'Église dans le monde. Vatican II a fait appel à l'insertion de l'Église dans le monde, au cœur de l'histoire, au sein de la société (Lumen Gentium 50, Gaudium et Spes 40). Cependant, l'Église en Amérique latine dira : l'insertion dans le monde ne suffit pas; elle est certes nécessaire, mais dans quel monde? Celui des minorités des inclus ou de la majorité des exclus? Celui dont $20 \%$ de la population possèdent $80 \%$ des ressources de la planète ou celui où $80 \%$ des exclus végètent avec les $20 \%$ des ressources restantes? Medellín $(14,10)$ nous avertit: la mission évangélisatrice, dans un continent marqué par l'exclusion, implique la dénonciation de toute injustice ou oppression présente dans la société, comme signal de contradiction envers les oppresseurs. La diaconie historique de l'Église, en tant que service prophétique face à de grands intérêts en jeu, peut aboutir à la persécution et au martyre, comme conséquence de la fidélité à l'option pour les pauvres (Comblin 2006, 301-304).

Le témoignage des martyrs des causes sociales est l'expression la plus vive du vécu de la foi chrétienne dans la fidélité à l'option pour les pauvres, 
dans une société injuste et excluante. Aparecida (98) parlera d'eux comme nos saints et saintes non encore canonisés.

\subsection{La théologie comme prophétie}

À son tour, la théologie comme intelligence reflétant des pratiques de foi des communautés ecclésiales insérées en perspective transformatrice au sein de la société ne peut être que prophétique (Ferraro 1996). La prophétie de la théologie passe par une théologie publique, articulatoire de la foi avec les grandes causes de l'humanité, qui sont les causes de l'Évangile même (Brighenti 2002). Elle passe également par une théologie, reflet de la préférence de Dieu pour les pauvres, dans une perspective œcuménique, interreligieuse, interculturelle, écologique, éthique, de genre ou de sexe, affinée avec les mouvements sociaux et les forces vivantes de la société.

Du point de vue ecclésial, une théologie prophétique dépend, d'un côté, des pratiques ecclésiales à perspective transformatrice et, de l'autre, de l'insertion du théologien dans une communauté ecclésiale concrète, sans parler du besoin d'espaces de liberté pour la création et l'exercice d'une réflexion critique. Du côté des pratiques, la prophétie de la théologie dépend de l'authenticité du mode d'être Église des communautés ecclésiales. Tant qu'il y aura des pauvres et un Évangile, la perspective prophétique et transformatrice de l'action ecclésiale sera une condition pour l'authenticité de l'Église elle-même. Une Église orpheline de société conduit à une théologie orpheline de pauvres, et donc orpheline d'Évangile, puisque l'option pour les pauvres s'enracine dans la foi christologique (Benoît XVI 2007). Du côté de l'interaction entre le ministère théologique et les pratiques ecclésiales et sociales, dans une perspective libératrice, le lieu du théologien et de la théologie dans l'académie sont remis en cause. Si la théologie est un "moment second ", l'intelligence réfléchie d'un «moment premier» que sont les pratiques ecclésiales à perspective transformatrice, l'éloignement du théologien de ces pratiques compromet la prophétie de la théologie comme expression authentique de la théologie elle-même, car celle-ci ne serait plus une contribution à une action ecclésiale anticipatrice de la présence du Royaume eschatologique dans la concrétude de l'histoire. Une théologie herméneutique-contextualisée ne peut perdre de vue son vrai lieu, qui est la communauté ecclésiale, insérée "par contraste» dans un contexte marqué par l'exclusion, en ses diverses nuances (Boff 1986, 27).

La prophétie de la théologie, en relation avec la société, rend hommage à son importance sociale, en son moment historique. La théologie est une 
des médiations ecclésiales privilégiées pour faire parvenir le message évangélique comme «bonne nouvelle» (horizon de vie, de sens, de salut) à la société, de par son autonomie vis-à-vis de l'Église. Cela implique une théologie publique, ayant comme interlocuteurs tous les sujets des différentes situations, avec lesquels l'action pastorale doit interagir (Comblin 2008). Aussi la théologie doit-elle être «compagne de route» des sciences, en relation inter et transdisciplinaire, et des Églises et des religions, en perspective œcuménique et interreligieuse. Elle doit être la "compagne de route» d'une humanité tout entière pérégrinante, syntonisée avec ses grandes causes, au sein de laquelle chemine aussi le peuple de Dieu. Une théologie chercheuse d'une vérité, dont elle est dépositaire, mais qui la dépasse infiniment (avoir la plénitude de la révélation ne signifie pas en avoir l'exclusivité ni en avoir tout saisi). Une théologie en dialogue avec les cultures, au sein desquelles se donnent les conditions de vie, notamment une production théorique incluant une réflexion théologique. Une théologie, enfin, qui soit la «compagne de route» surtout des exclus de la société et qui doit être juste et solidaire, afin d'être signe de la présence du royaume, expression des desseins de Dieu pour l'ensemble de l'œuvre de la Création.

\section{Altermondialisation et théologie}

Les points discutés dans les deux sections précédentes nous donnent l'horizon nécessaire pour présenter maintenant, dans les grandes lignes, les éléments qui caractérisent l'altermondialisation dans la réflexion théologique au Brésil. Pour des raisons d'espace, nous nous limiterons à trois sujets:

1) l'horizon utopique d'un "autre monde possible";

2) une société civile mondiale en tant que nouveau nom de l'espoir solidaire;

3) les bases d'un autre monde possible.

Au Brésil, des théologiens de l'économie comme Hugo Assmann ou son disciple Jung Mo Sung, de l'écologie comme Leonardo Boff, et de l'éthique sociale comme J. Comblin ou J. B. Libânio font partie de ceux qui ont le plus réfléchi à la question, sans oublier la contribution de théologiens et de biblistes d'autres Églises, comme Milton Schwants et Válter Altemann. Dans ce qui suit, nous nous inspirerons de leurs contributions à la recherche et en vue de l'édification d'un monde juste et solidaire.

La thèse sous-jacente est que la situation actuelle d'exclusion et de pauvreté de larges secteurs de la population de nos pays fait émerger, avec réa- 
lisme et cruauté, un modèle économique concret, qui a créé l' «ordre international » actuel, héritage du système colonialiste et qui se perpétue à travers la globalisation et le marché global. Il s'agit d'un darwinisme social (Sebastián 1989, 7), qui exalte les plus doués et les plus qualifiés à la compétition économique, au nom d'un "progrès" qui, sous prétexte de construire un ciel sur terre, condamne les deux tiers de l'humanité à un enfer de pauvreté et de misère, véritables orphelins de l'actuel processus de la globalisation (Boff 1996).

Des points de vues politique et économique, une solution radicale passe par l'établissement d'un nouvel ordre international, base d'un développement intégral et solidaire qui peut promouvoir la vie et le respect de la nature, cette dernière étant l'origine des ressources, une sorte de «maison de tous ». Les problèmes du monde ne pourront être réglés qu'en faisant une analyse à partir d'une perspective globale (Iriarte 1992, 143-144). Mais serait-ce là une solution définitive? N'y aurait-il pas un problème culturel à la base du modèle politique et économique en vigueur, modèle qui soutient l'ordre économique international actuel? En réalité, créer un nouvel ordre mondial tel que nous le décrivons implique plus que de simplement changer les structures; ce projet implique le défi de créer une culture de la solidarité en tant que dépassement de la mentalité de colonisés et en substitution à l'actuelle logique d'exclusion. Mais alors, une autre question se pose: sur quelle base cette culture doit-elle être fondée? Sur quel absolu pourrait-on poser les bases de valeurs comme la solidarité, le respect et la promotion de la dignité humaine, la communication universelle des biens, etc. ? Et, plus encore, quel pourrait être l'horizon de sens de ces valeurs? Aujourd'hui, la sphère du religieux est de plus en plus montrée du doigt à cet égard (Sung 2008).

Dans cette perspective, Hans Küng (1992) a mis en évidence que la survie de l'humanité dépend de la création d'un ethos fondamental pour tous les hommes et toutes les cultures, ce qui est impossible, selon lui, si les grandes religions ne parviennent pas à des normes, des valeurs, des idées et des buts fondamentaux communs.

\subsection{L'borizon utopique de la solidarité}

Un «autre monde» devra être nécessairement solidaire. Et l'édification d'un monde solidaire passe par la gestation d'une culture qui lui apporte son soutien, sous peine de se réduire à une chimère. C'est la fameuse «fin de l'histoire» (Fukuyama 1992), avec la conséquente crise des utopies qui 
nous rend sceptiques quant aux possibilités de l'espoir en tant qu' «inédit viable» (Freire 1993).

Ce monde, de plus en plus excluant, est la radiographie et la claire attestation de la vigueur d'une culture-non-solidaire, régnant dans la civilisation occidentale et présente surtout dans une mentalité colonisatrice qui persiste. Les affirmations fallacieuses du marché, telles que l'assurance de trouver dans le marché total un chemin sûr vers le bien-être de tous, l'écroulement du socialisme, la «fin de l'histoire», etc. (Assmann 1997), ont contribué au climat actuel d'anti-utopisme dans lequel la «dictature du présent » paraît être le seul horizon possible. La grande masse des exclus de ce monde arrive au début du troisième millénaire, non seulement plus pauvre, mais également en perte d'espoir.

\subsubsection{Une culture d'anti-solidarité comme négation d'un ethos fondamental}

Les faits socioculturels les plus importants de cette fin/début de millénaire sont: l'accroissement vertigineux du nombre d'humains «excédentaires » ou «de trop » à l'intérieur du système de globalisation via le marché total et la destruction de la nature. Ce sont les deux thermomètres qui mesurent le degré de culture d'anti-solidarité entre nous.

L'exclusion, d'abord, est assujettie à un progrès qui n'est pas pour tous. Le système peut uniquement garantir la vie de ceux qui sont nécessaires au développement du marché. C'est le passage du "capitalisme utopique ", qui croyait pouvoir satisfaire aux nécessités de tous, au «capitalisme réaliste », dont la finalité est l'efficacité du marché et le profit maximal. Ceux qui ne font pas partie du système ne comptent pas. Ceux-ci non seulement augmentent le surnombre des exclus, mais ils sont également vus comme un obstacle à l'optimisation du marché. Ils sont jetables, dans la mesure où leur mort n'affecte pas l'efficacité du système mais, au contraire, contribue à son perfectionnement. Les pauvres sont un obstacle au progrès, les ennemis du marché parfait, victimes coupables dont le sacrifice est nécessaire afin de sauver le système. Leur «liquidation » n'est donc pas un problème moral, mais simplement une question de «nettoyage social». On passe de l'idéologie de la sécurité nationale à l' "idéologie de l'exclusion» (Assmann 1997).

Le modèle économique de marché actuel se base sur des mécanismes d'échange qui génèrent un monde de riches et un sous-monde de pauvres (Vitoria 1999, 6). Il s'agit d'une logique qui dérive d'une «éthique de mar- 
ché », ou plus exactement d'une anti-éthique, antagonique à la promotion de la solidarité. Dans cette logique, les moyens - ressources technologiques, scientifiques et financières - deviennent les seules fins utiles. L'efficacité et la compétitivité y semblent être des valeurs absolues, sans prendre la vie humaine et ses écosystèmes en considération, ni le soin de la nature. L'économie, dépouillée de sa condition de moyen de promotion de la vie, est promue comme fin en elle-même, ce qui, dans la pratique, signifie sa réduction à la logique de la compétitivité, du calcul utilitariste et du profit maximum. Toute orientation vers le bien commun ou vers la construction d'un monde commun disparaît. Il y a, par rapport aux biens, une primauté du pouvoir d'échange sur le pouvoir d'usage, qui mène à une mercantilisation des relations humaines en général.

À l'intérieur de la culture de non-solidarité, générée ou alimentée par le système actuel, le marché total est la société parfaite et la technologie apparaît comme le messie qui apporte le salut à toute l'humanité. La défense de la vie et de la nature est vue comme un obstacle au développement, étant donné que le système fonctionne à condition de relativiser la solidarité. D'où sa logique sacrificielle.

Le second thermomètre qui mesure le degré du manque de solidarité entre les peuples est la destruction de la nature. Dans le système actuel, l'écologie ne doit pas ralentir l'efficience du marché. L'utilisation des ressources naturelles suit la même tendance que la valeur de l'efficience: une exploration maximale pour une utilité marchande maximale. Conserver la nature est un obstacle à la logique du marché. Même la biodiversité apparaît comme une bonne affaire, même si on détruit en un jour ce que la nature a mis des millions d'années à construire. Pourquoi économiser la nature, si elle est aujourd'hui une source de profit? Demain, si demain il y a, la technique fournira d'autres moyens de ressources! Il s'agit d'une économie de rapaces, incapable d'un développement durable. Celle-ci oublie que les ressources et les biens de la création ont été donnés pour tous, pour le bien de tous, de tous les temps. Elle ne tient pas compte de l'impératif d'une cohabitation harmonieuse de l'être humain avec son environnement, au risque de mettre en péril la viabilité de la vie et de la planète (Boff 2001).

Nous sommes donc dans un système qui colonise jusqu'à l'éthique culturelle. En se basant sur le «mythe du progrès économique », il fait de l'argent et du profit le moteur de l'histoire, plus exactement, d'une histoire inhumaine. L'argent, en tant que valeur d'échange, ayant pour fin de garantir la satisfaction des nécessités vitales, devient un dieu, une fausse idole, qui va jusqu'à justifier des sacrifices humains, à l'intérieur d'un «ordre nécro- 
phile» (Vitoria 1999, 9). Les institutions juridiques des États et des organisations supra-étatiques, plutôt que d'être des instances de défense et de promotion du bien commun, fonctionnent comme des instances de globalisation du marché, afin de faciliter les flux de marchandises et de capitaux, plus subventionnés que le social. En résumé, le système "présuppose une mystique cruelle de la performance et du culte à l'efficience" (Sung 1997, 12, nous traduisons), ce qui en fait non seulement un système "économiciste ", mais également un système qui est tributaire d'un "fondamentalisme économique» (Sebastián 1989, 102) ou d'un "intégrisme économiciste». Sous prétexte de «modernisation », on affirme le caractère inévitable des processus en cours et on accuse de "gauchistes romantiques » ceux qui refusent d'accepter que «l'état naturel de la société [soit] le marché» (Vitoria 1999, 11 , nous traduisons).

\subsubsection{La culture de la solidarité en tant qu'ethos fondamental}

Bien que la culture de la solidarité ne puisse construire d'elle-même une alternative au système colonialiste de marché, elle possède la force de se constituer en une alternative à l'esprit du système, plus concrètement, en sa base culturelle. Le système du marché total, qui exclut les majorités et détruit la nature, a pour âme une «culture », une "éthique» et une "spiritualité » qui doivent être substituées par une culture de la solidarité (Sung 2005).

Par ailleurs, il n'est pas ici question de diaboliser le marché en tant que tel. En premier lieu, celui-ci ne se limite pas à la dimension économique compétitive, il est avant tout un symbole des virtualités démocratiques, en tant que mécanisme d'échange entre les communautés, les peuples et les cultures. Il ne fait aucun doute que le marché, en tant qu'institution, est un outil indispensable dans la lutte contre la pauvreté, afin de rendre possible une vie digne pour tous. Un deuxième aspect concerne le totalitarisme d'un marché transformé en une fin en soi, en un dieu et basé sur des fourberies aux accents quasi religieux, telles que: le marché mène à une croissance économique infinie (alors qu'elle a ses limites); si l'économie croît, alors le niveau de vie de tous s'améliore (or, le chômage et la pauvreté peuvent être des conséquences de cette croissance); si chacun lutte pour lui-même, tout le monde progresse (en réalité, les plus faibles disparaissent); le marché permet une meilleure distribution des biens et des prix plus justes (mais dans une relation entre inégaux, les plus forts ont toujours l'avantage); le futur est assuré lorsque les problèmes immédiats sont résolus (alors qu'ar- 
rêter de faire des projets pour le futur peut signifier sa non-viabilité); le marché doit profiter au maximum des richesses de la nature (alors que ne pas prendre en compte les limites de la nature, c'est rendre la vie impossible); etc. De tels principes n'ont fait que rendre les riches - des pays riches ou pauvres - encore plus riches.

D'un point de vue culturel, le projet de solidarité suppose un engagement, afin de surpasser la prédominance du paradigme fonctionnel colonisateur de la culture occidentale. À l'instar de Habermas, Rawls, Ricoeur ou Levinas, il est logique d'argumenter sur cette responsabilité mutuelle et cette co-implication des uns envers les autres, pour une question de survie, mais encore plus pour une histoire digne de l'être humain, celui-ci étant capable de la planifier dans toutes ses potentialités (Mardones 1994, 42). Non pas que le pragmatique, l'efficace, l'utile, l'avoir, entre autres, n'aient aucune valeur. Il s'agit, avant tout, de situer ces derniers sur une échelle de valeurs, en partant du fait que d'autres aspects de la rationalité humaine doivent avoir la primauté. On ne peut pas confondre des moyens tels que le marché, la marchandise, les ressources financières, ou encore l'avoir, avec des fins, comme la défense et la promotion de la vie, la convivialité fraternelle, la solidarité et, ultimement, l'être lui-même. L'urgence d'une culture solidaire se présente, aujourd'hui, comme une alternative au fonctionnalisme qui envahit tous les contextes de la vie. Sans cet esprit de solidarité, il est impossible de produire la culture correspondante (Assmann 2006).

Le passage d'un esprit solidaire à une culture de la solidarité exige un style de vie correspondant. Entre autres choses, le plus fondamental est de retrouver le sens d'une vie austère, face à une société de consommation, austérité qui, de façon prophétique à travers le témoignage, dénonce la relativisation de l'absolu légitime et l'absolutisation indue du relatif, et annonce des valeurs plus définitives telles que la solidarité, la gratuité, la vie intérieure, etc. Il ne peut y avoir de culture de la solidarité tant que règne la mercantilisation des relations, tant que les États sont régis par des intérêts nationalistes ou privés, tant que les riches minorités ne se résignent pas face au réalisme-sans-futur des majorités, tant que, enfin, nous ne remplaçons pas un certain modèle de bonheur basé sur l'hédonisme et le nonengagement vis-à-vis des autres. Si ne nous parvenons pas à matérialiser la solidarité dans des formes de vie ou dans des habitudes de tout un peuple, la transcendance et les significations politiques de ce dernier seront frustrées (Alvarez Bolado 1991, 42-43). 
En fait, la culture de la solidarité part d'un fait de conscience: la différence entre être et avoir. C'est uniquement lorsque sont établis une relation hiérarchique et un équilibre exigés par la nature humaine elle-même entre l'être et l'avoir que l'on est capable de juger inhumain l'avoir de certains au prix du non-être de tant d'autres. C'est alors qu'il sera possible de comprendre que, bien que le développement implique des aspects économiques et sociaux, s'il ne dispose pas à sa base d'une culture de la solidarité ou du respect envers l'altérité, il sera sa propre ruine, ainsi que celle de son milieu. Une personne est toujours une fin en soi, jamais un moyen. Néanmoins, une telle conscience passe par l'expérience de la dignité envers soi-même, ce qui requiert une stimulation prophétique des lieux d'apprentissage et de légitimation.

\subsection{Une société civile mondiale comme nouveau nom de l'espoir solidaire}

Une culture de la solidarité ne saura réaliser l'idéal utopique de la fraternité que si elle rejoint la politique, basée sur une société civile mondiale. Aujourd'hui, celle-ci est le domaine obligatoire capable de canaliser l'organisation de la collectivité en vue d'un autre monde. Depuis les Grecs, la politique a été entendue comme un mode pratique pour donner une forme sociale aux exigences éthiques. Le grand problème du monde de nos jours, face à la pauvreté, n'est pas que l'on ne sache pas quoi faire, ni comment. Le fonctionnement des économies est connu. Nous savons comment éliminer la pauvreté, comment distribuer les revenus. Les groupes «économiquement inutiles » connaissent, de façon générale, leurs droits. La conscience existe; ce qui fait défaut, c'est le pouvoir-faire. Or, la politique, fondée sur une société civile mondiale, a ce pouvoir (Arruda 1999).

De nos jours, cependant, l'exercice utilitariste de la politique a contribué à son désenchantement. Qu'elle soit exercée dans des systèmes autoritaires ou dans des partis démocratiques, la politique s'est transformée en un simple "art du possible», dépourvue de toute référence éthique. Elle est passée du soin de la "polis", chez les Grecs, à une stratégie de prise de pouvoir; une fois celui-ci acquis, la politique devient la gérante d'intérêts privés? qu'il s'agisse d'individus ou de groupes d'intérêts. Cependant, nous attendons de la politique plus qu'un «art du possible »: qu'elle soit un art de rendre possible ce qui est apparemment impossible. Cela suppose un ré-enchantement de celle-ci, à travers la récupération de son orientation éthico-solidaire. Les solutions politiques actuelles, à l'intérieur des modèles 
politiques de la démocratie libérale, sont incapables de canaliser les aspirations éthiques de la collectivité. Une redéfinition du rôle de la vie parlementaire, des partis, des syndicats, des mouvements sociaux, etc., est urgente face à l'impératif du soin et de la promotion de la "polis». Ces exigences vont d'un approfondissement des idéaux démocratiques jusqu'aux paramètres de la démocratie libérale ou représentative, en passant par le besoin d'honnêteté et de transparence de la part de ses militants, afin que ces moyens se constituent en mécanismes capables de canaliser la participation responsable au niveau planétaire (Mardones 1994, 44).

\subsection{Les bases d'un développement intégral et solidaire}

Une culture de la solidarité et un ré-enchantement de la politique, en tant que pouvoir de réalisation de la solidarité à travers une société civile mondiale, rendent plus effectives la recherche et l'édification d'un «autre monde ", un monde fondé sur un développement solidaire. Ses bases reposent sur certains principes de base (Camacho 1992).

\subsubsection{Un développement qui dépasse le concept actuel de développement}

Le point de départ, dans l'édification d'un "autre monde », est le dépassement du modèle actuel de développement. Le modèle de développement et de société, actuellement dominant et en crise, a ses racines dans le système économique libéral, construit à partir d'un point de vue du Nord, en ses diverses versions colonialistes et néocolonialistes dans les pays du tiersmonde. Du point de vue dominant, ce qui est vu comme une grande épopée européenne depuis les dernières 500 années a été, dans l'optique du Sud, une grande tragédie marquée par des invasions, des explorations, la dépendance, l'esclavage, la négation de l'autre, etc. Malgré le phénomène de globalisation, les anomalies entre l'hémisphère Nord et l'hémisphère Sud persistent.

Il s'agit d'un modèle de développement pervers, fondé sur de profondes injustices structurelles, dans lesquelles les nouvelles technologies créent une distance de plus en plus grande entre le Nord et le Sud. La preuve en est qu'aujourd'hui, le Nord, qui compte un quart de la population mondiale, consomme les trois quarts des richesses mondiales, alors que les peuples du Sud ne consomment qu'un quart de celles-ci. Cela s'explique uniquement par les mécanismes de drainage des ressources du Sud vers le Nord (Regidor 1992, 29). Les conséquences sont dramatiques: outre la dette financière, il 
y a également une dette éthico-historique, contractée par des peuples du Nord, à cause de la négation de la subjectivité, de la culture et de la religion des peuples du Sud. Néanmoins, ce type de modèle de développement n'est pas universalisable. Il ne suffit pas non plus de chercher des modes d'ajustement du modèle actuel de développement en réduisant au minimum son impact social et écologique. Il faut en venir au problème de fond qui nous éclaire sur ce qui doit être produit, pour qui et comment. Le concept que l'on nomme "développement durable " s'insère généralement dans le cadre de l'idéologie de la croissance économique illimitée, dans laquelle ce qui doit réellement être «durable» est le développement. Dans cette perspective, est également inséré ce que l'on appelle "écodéveloppement", dans la mesure où ce qui est recherché est une croissance économique sans contaminer la nature, mais en accordant tout de même la priorité à la croissance, selon le modèle actuel de développement. Toutefois, pour rembourser ou restituer ce qui appartient aux peuples et à la nature, il faut changer radicalement le modèle actuel de développement, en rejetant l'idéologie de la croissance.

Le concile Vatican II définit, dans Gaudium et Spes, le développement authentique selon deux exigences:

1) qu'il soit intégral, c'est-à-dire qu'il réponde non seulement aux nécessités économiques et matérielles, mais également aux besoins culturels, affectifs, éthiques et spirituels de l'être humain;

2) qu'il soit solidaire, c'est-à-dire qu'il s'étende à toutes les personnes et à tous les peuples (Gaudium et Spes, 417-418).

Dans Populorum Progressio (1967), repris par Benoit XVI dans Caritas in Veritate, Paul VI insiste, lors de la caractérisation du sous-développement comme sous-produit du développement des pays riches, sur l'obligation des pays industrialisés d'aider les pays les plus pauvres, afin de compenser pour les comportements injustifiables des temps passés, et sur la nécessité de construire un ordre international basé sur la justice, étant donné que "le développement est le nouveau nom de la paix».

Enfin, la radicalité du changement dans le modèle de développement et dans le modèle de société consiste en la suppression du principe du profit, ainsi que de l'idéologie de la croissance économique illimitée. Ces deux facteurs sont les piliers fondamentaux du système actuel, basé sur des comportements de l'ordre de la domination, de l'exclusion de peuples entiers et de l'exploitation sans limites de la nature. À partir d'une base éthique, il s'agit de forger un modèle de développement qui promeut la justice sociale 
et la justice écologique au même titre que l'établissement des changements structuraux dans la production, la consommation et la distribution.

\subsubsection{L'autonomie des peuples et leur droit à l'autodétermination}

En deuxième lieu, un développement juste et solidaire implique l'autonomie des peuples et leur droit à l'autodétermination au sein d'une vraie communauté internationale. Aujourd'hui, le statut de citoyen universel est occupé par le grand capital. La globalisation, dans les modèles actuels, impose des limites à la souveraineté nationale et à la capacité d'action des États. Les économies nationales sont, plus que jamais, en dépendance croissante des dynamiques de l'économie globale dans les secteurs commercial, financier et monétaire. Il y a une réduction du degré d'indépendance nationale dans la réalisation d'une politique autonome, qui contribue à l'affaiblissement progressif des fonctions de l'État en ce qui concerne la redistribution des apports, ainsi que son rôle de modérateur dans les tensions sociales (Arruda 1999, 107-108). Au nom d'une économie de marché ouverte, les macro-projets des nations les plus pauvres deviennent irréalisables, à mesure que les pays riches ne cachent plus leur protectionnisme, qui leur fut une mesure utile, tant dans le passé ? à l'ère de l'industrialisation - qu'aujourd'hui, encore afin de protéger leurs industries et leurs marchés. Le marché, à lui seul, ne peut pas éradiquer la pauvreté, ne promeut pas le développement humain ni social. En plus, il protège encore moins l'environnement. Bien que toute l'économie nationale fasse partie de l'économie mondiale, sans un ordre international juste, il n'y aura pas de développement juste et solidaire.

Dans l'édification d'un "autre monde" où tous et chacun puissent avoir leur place, il est fondamental que des relations symétriques s'établissent entre tous les peuples de la planète et entre les différentes cultures, exprimées dans la reconnaissance, d'une part, de la subjectivité des peuples et, d'autre part, de la relativité et des limites de toute culture. Les cultures ancestrales méritent un respect tout particulier, elles qui sont une réserve de nouvelles valeurs, de modes de vie et de coexistence, une inspiration dans la recherche et l'édification d'un monde solidaire.

\subsubsection{La participation de tous les peuples aux sujets internationaux, sur un pied d'égalité}

Un autre monde, où tous et chacun ont leur place, exige la participation de tous les peuples aux enjeux internationaux, et ce, sur un pied d'égalité, 
condition pour la bonne correction du fonctionnement des institutions qui implique le bien-être de tous, des institutions aujourd'hui gouvernées selon les intérêts des plus puissants. L'ONU elle-même, de nos jours, répond encore aux intérêts des pays les plus riches ou des pays vainqueurs de la Seconde Guerre mondiale. Bien qu'elle soit l'institution multilatérale la plus universelle, elle ne joue pas de rôle dans les processus réels de négociation et de prise de décisions sur des thèmes vitaux comme celui du développement des peuples.

La mondialisation de la planète doit être gouvernée, non pas par un pouvoir supranational, mais par la concertation de la communauté des nations, évitant ainsi les distorsions actuelles. Sans la participation paritaire de tous les pays, il ne sera pas possible de corriger le désordre international actuel. Étant donné la conjoncture économique actuelle, il est urgent d'établir une organisation internationale dotée de pouvoirs permettant de régulariser le système global financier et d'établir des normes de contrôle de la spéculation (Arruda 1999, 112) — particulièrement des moyens financiers toujours à la recherche de meilleurs marchés, dans le modèle d'une "économie de casino » - , soit un système régulateur global, ainsi que des institutions juridiques globales, avec le pouvoir d'imposer certaines règles éthiques dans le système financier.

\subsubsection{La destinée universelle des biens de la planète}

Un autre monde, où tous et chacun ont leur place, doit être basé sur le principe de la destinée universelle des biens de la planète. Un développement solidaire implique de mettre la totalité des ressources à la disposition du bien-être de tous, ce qui exige une participation efficace et équitable dans la distribution des ressources naturelles et des biens manufacturiers. Cela suppose une correction des mécanismes «spontanés ", mieux encore, déterminés pour des grands intérêts de groupes ou de pays qui régulent actuellement les échanges économiques et financiers.

L'établissement de justes prix des matières premières, principale source de richesse des pays les plus pauvres, par rapport aux autres produits manufacturiers, est un élément fondamental de cette correction. Sans cela, il n'est pas possible de distribuer de façon équivalente les biens de la planète. Tout appartenant à tous, selon le plan créateur de Dieu, il n'est donc pas juste que certains vivent dans le superflu à côté d'une majorité qui ne possède pas le nécessaire. Pour cela, il faut passer de principes acceptés par tous ou presque à la pratique, dans la création d'institutions qui garantissent la réalisation de ces idéaux. 


\subsubsection{Une option préférentielle effective pour les peuples les plus pauvres}

Il n'est pas possible de construire un autre monde possible, basé sur un développement solidaire, sans un ordre économique qui offre une option préférentielle effective pour les peuples les plus pauvres. Il faut faire face au défi de l'élimination des inégalités qui ont été produites entre les diverses régions du globe, conséquences d'un ordre injuste et maintenu en vigueur depuis des siècles. Cela exige la canalisation des ressources spécifiques aux peuples les plus pauvres, en redistribuant les ressources et le revenu mondial. Les riches, individus, pays ou hémisphères, ne peuvent continuer à devenir de plus en plus riches, aux dépens des pauvres de plus en plus pauvres. Si tout appartient à tous, alors, au minimum, le superflu appartient à celui qui ne possède pas le nécessaire. Le niveau de consommation des peuples de l'hémisphère Nord est un affront à la misère des grandes majorités des peuples du Sud.

Un plan global de soutien efficace envers les pauvres ne serait d'ailleurs pas inédit dans l'histoire. Le plan de reconstruction de l'Europe, en particulier de l'Italie et de l'Allemagne en période d'après-guerre - et qui a résulté en un boom économique à partir des années 1960 -, est un fait connu de tous. Même chose lors de l'unification de la Communauté européenne.

\subsubsection{La conscience que nous formons une communauté mondiale unique}

Un autre monde, où tous et chacun ont leur place, correspond à la conscience du destin commun de tous les peuples de la planète, lesquels forment une communauté mondiale unique. Dans ce monde, il ne peut y avoir de nationalismes étroits qui dénigrent la dignité humaine des citoyens universels que nous sommes. Nous sommes en train de faire une dure expérience qui prouve de plus en plus que les problèmes régionaux sont également des problèmes mondiaux et qu'ils affectent donc tout le monde. Il n'est désormais plus possible de construire sa propre arche, ni de penser à des projets basés sur les privilèges de certains (Boff 1994).

La destruction de la planète par une économie de rapaces qui épuise les ressources naturelles et qui chosifie l'être humain, impose le défi d'un modèle de développement basé sur le soin de la vie, de la planète, de l'être humain et de toute l'œuvre de la création. Pour cela, nous avons été conçus 
comme des créatures-créatrices. D’un côté, le modèle hégémonique inspiré de la philosophie et des sciences modernes est totalement inviable (de Bacon à Galilée, de Descartes à Newton). C'est un modèle basé sur un anthropocentrisme narcissique: l'être humain est le centre, le seigneur du monde et le maître de la nature, si bien qu'il peut la consommer comme un objet, inexorablement. Dans ce modèle de relation, les autres êtres, animés ou inanimés, n'ont pas de valeur en soi. La réalité est vue comme un mode de production et de consommation, dans lequel la priorité est pour le profit, l'industrialisme et l'idéologie du progrès au sens de croissance économique illimitée. Le plus grave, dans la justification du comportement de domination, de possession et de violence contre la nature, c'est l'attitude de négation de l'altérité et de la différence de l'autre. L'autre n'est, également, qu'un «capital humain» à exploiter.

À l'opposé, un biocentrisme réductionniste (Regidor 1992, 40-41), pour lequel la vie des animaux et des plantes est le centre de tout, est également incompatible avec la recherche et l'édification d'un autre monde où tous et chacun ont leur place. Ce modèle est centré exclusivement sur l'attention envers les espèces vivantes en danger d'extinction et oublie que les êtres humains font également partie de l'écosystème. On en arrive au point d'accuser les êtres humains de constituer un préjudice à l'équilibre de la biosphère. On ne prend pas en compte la différence, la valeur et le rôle de l'être humain sur la planète et, en conséquence, on n'arrive pas à déterminer la responsabilité de celui-ci dans l'histoire. On ne s'aperçoit pas de l'existence d'une relation intrinsèque entre la dévastation de la nature et la dévastation des peuples et de leurs cultures. Il n'est pas rare que ce biocentrisme réductionniste soit lié à un certain type de romantisme écologique, qui perd de vue la centralité de la valeur et de la dignité de la vie humaine par rapport à son écosystème, comme si la vie des êtres humains constituait seulement une partie de l'écosystème, à côté du reste.

Un autre monde, où tous et chacun ont leur place, doit être basé sur la reconnaissance et le respect réciproque de la valeur intrinsèque et du rôle de tous les êtres de l'écosystème. Il faut partir du principe que l'être humain n'est pas séparé de la nature ni supérieur aux autres êtres: ni centre ni propriétaire. Au contraire, il fait partie de la nature, de l'écosystème, de la planète. Cependant, il se distingue des autres êtres de l'écosystème par sa capacité morale, qui en fait un être avec des responsabilités. Il est celui qui est responsable de l'administration et du soin de la planète, non en tant que propriétaire. Cette responsabilité se concrétise par le service des autres 
êtres, service ou rôle ayant des limites et des lois pour son équilibre. Tous les êtres de l'écosystème ont une valeur intrinsèque, une différence et une altérité qui ne proviennent pas de l'être humain, même si ce dernier est le seul à les reconnaître. Bien que, à travers son rôle d'agent moral et responsable de la planète, l'être humain soit une fin et non un instrument, il n'est pas fin ultime ni centre de tout, et les êtres de la nature n'existent pas prioritairement pour l'être humain. Tous les êtres, incluant les êtres humains, appartiennent à la magnanime œuvre de la Création du Père, dans l'Esprit, rachetée par son Fils (Boff 2001).

\section{Pistes de conclusion}

Nous nous trouvons dans une période de crise d'un vieux monde. Un nouveau monde est urgent et nécessaire. Pour que ce dernier soit possible, il faut faire de cette période une période pascale, de passage, ce qui implique d'être habité par l'espoir. Non par un espoir passif, miraculeux et momentané, mais par un espoir actif, capable de se ré-enchanter avec l'utopie d'un monde juste et solidaire, où tous et chacun ont leur place.

Par le passé, face à l'avènement du projet de la civilisation moderne, l'Église a assumé une posture apologétique, diabolisant les valeurs évangéliques de la modernité, uniquement parce que celles-ci se situaient en opposition à certains vieux usages ou vieilles doctrines, pourtant dépassés, de l'Église. Si celle-ci avait respecté et accueilli l'œuvre de l'Esprit dans l'histoire, au-delà des murs ecclésiaux, elle aurait eu la possibilité de contribuer à éviter beaucoup des anti-valeurs de la modernité, maintenant en crise.

Néanmoins, la crise actuelle de la modernité est essentiellement due aux nouvelles réalités et aux valeurs émergentes, plutôt qu’à ses équivoques, aussi nombreuses et néfastes furent-elles. Le nouveau monde est déjà en gestation, dans les multiples initiatives individuelles du réseau de gens et d'organismes qui engendrent un mode de vie basé sur la coopération, neutralisant ainsi la compétition, et sur la solidarité, ce qui disqualifie le cynisme des satisfaits. Les chrétiens doivent s'unir autour de ce «nouveau vin» et contribuer efficacement à la construction d'un «ordre nouveau», d'un «autre monde», urgent et nécessaire, afin qu'il soit la «maison de tous". 


\section{Références}

Alvarez Bolado, A. (1991), « Giro del siglo» y solidaridad, Maliaño/Bilbao, Sal Terrae (Aqui y Ahora 24).

Arruda, M. (1999), Dívida E(x)terna. Para o capital, tudo; para o social, migalhas, Petrópolis, Vozes.

Assmann, H. (1997), Las falacias religiosas del mercado, Barcelona (Cuadernos Cristianisme i Justícia 76).

- (2006), Competência e sensibilidade solidária. Educar para a esperança, Petrópolis, Vozes.

BENOÎT XVI (2007), «Discours Inaugural », $V^{e}$ Conférence générale de l’Épiscopat Latino-américain et des Caraïbes, Aparecida (Brésil).

Bigo, P. et F. B. de Ávila (1986), Fé cristã e compromisso social, São Paulo, Paulinas.

Boff, C. (1990), «Epistemología y método de la teología de la liberación », dans Ellacuria et J. Sobrino, dir., Mysterium liberationis, Conceptos fundamentales de la teología de la liberación. I-II, Madrid, Trotta, p. 79114.

Boff, L. (1986), Teología desde el lugar del pobre, Santander, Sal Terrae.

- (1993), Ecologia, mundialização, espiritualidade, São Paulo, Ática. (1994), Nova era. Civilização planetária, São Paulo, Ática.

Rocco.

(1995), Ecologia. Grito da Terra, grito dos pobres, Rio de Janeiro,

(1996a) Brasa sob cinzas. Estórias do anticotidiano, Rio de Janeiro/São Paulo, Record.

Ática.

(1996b), Ecologia, Mundialização, Espiritualidade, São Paulo,

(2000), La dignidad de la tierra. Ecologia, mundialización, espiritualidad. La emergência de um nuevo paradigma, Madrid, Trotta.

(2001), Cuidar la tierra. Hacia uma ética universal, Mexico, Dabar.

Boff, L. et C. Boff (1986), Como fazer teologia da libertação, Petrópolis, Vozes/IBASE.

BRIGHENTI, A. (2002), «La mundialización de desafíos hasta entonces continentales. Interpelaciones para la inteligencia de la fe cristiana desde Brasil », Concilium, 296, p. 419-426. 
Camacho, I. (1992), "Economía y Moral », dans M. VIDAL, Conceptos fundamentales de ética teológica, Madrid, Editorial Trotta, p. 743-745.

Comblin, J. (2005), Quais os desafios dos temas teológicos atuais, São Paulo, Paulus.

(2006), «Los pobres en la Iglesia latinoamericana y caribeña», dans Amerindia, Tejiendo Redes de Vida y Esperanza. Cristianismo, sociedad y profecía en América Latina y El Caribe, Bogotá, IndoAmerican Press, p. 289-305. (2008), A profecia na Igreja, São Paulo, Paulus.

FERRARO, B. (1996), «Função da Teologia em meio a crises de referências, de utopias e de esperança ", dans M. FABRI Dos Anjos, dir., Teologia e novos paradigmas, São Paulo, Soter-Loyola, p. 177-189.

Freire, P. (1993), Pedagogía de la esperança. Un reencuentro con la Pedagogía del Oprimido, Madrid, Siglo XXI.

Fukuyama, F. (1992), O fim da história e o último homem, Rio de Janeiro, Rocco.

IRIARTE, G. (1992), La deuda externa es inmoral. La deuda externa como problema ético de proporciones universales, Bogotá, Paulinas.

KüNG, H. (1992), Proyecto de un ethos mundial, Madrid, Casterman.

Mardones, L. M. (1994), Por una cultura de la solidaridad. Actitudes ante la crisis, Maliaño/Madrid, Cuadernos Fe y Secularidad/Sal Terrae.

MuÑoz, R. (2006), "Para una eclesiología latinoamericana y caribeña », dans Amerindia, Tejiendo Redes de Vida y Esperanza. Cristianismo, sociedad y profecía en América Latina y El Caribe, Bogotá, IndoAmerican Press, p. 333-352.

Palácio, C. (2000), «Trinta anos de teologia na América Latina. Um depoimento ", dans L. C. Susin, dir., O mar se abriu. Trinta anos de teologia na América Latina, São Paulo, Soter/Loyola, p. 51-64.

Regidor, J. R. (1992), «Ressarcir os Povos e a Natureza. Em busca de uma reconversão sócio-ecológica da sociedade ", Revista Eclesiástica Brasileira, 205, p. 23-44.

SAlgado, S. ( 2001), Exxodus, São Paulo, Record.

SEBASTIÁN, L. (1989), La gran contradicción del neoliberalismo moderno. O la sustitución del humanismo liberal por el darwinismo social, Barcelone (Cuadernos Cristianisme i Justícia 30). (2001), Mundo rico, mundo pobre, Santander, Sal Terrae. 
Sobrino, J. (2006), «El reino de Dios anunciado por Jesús. Reflexiones para nuestro tiempo ", dans Amerindia, Tejiendo Redes de Vida y Esperanza. Cristianismo, sociedad y profecía en América Latina y El Caribe, IndoAmerican Press, Bogotá, p. 267-288.

SousA SANTOS, B. de $\left(2009 \mathrm{a}^{2}\right)$, «The Word Social Forum: Toward a CounterHegemonic Globalisation », dans J. SEN et P. WATERMAN, dir., World Social Forum Challenging Empires, Montréal, Black Rose Books, p. 191204.

- $\left(2009 b^{2}\right)$, «The World Social Forum and the Future», dans J. SEN et P. Waterman, dir., World Social Forum Challenging Empires, Montréal, Black Rose Books, p. 378-386.

Sung, J. M. (1997), «Deseo mimético, exclusión social y cristianismo », Pasos, 69, p. 8-28.

(2005), Sementes de esperança. A fé em um mundo em crise, Petrópolis, Vozes.

(2008), Cristianismo de libertação. Espiritualidade e luta social, São Paulo, Paulus.

Susin, L. C. (2005), "Le danger de vivre et la créativité de la foi », dans H.A.M. Muller et D. VILLEPELET, Risquer la foi dans nos sociétés. Églises d'Amérique latine et d'Europe en dialogue, Paris, Kartala, p. 31-51.

Vitoria, J. (1999), Un orden económico justo, Barcelone (Cuadernos Cristianisme i Justícia; 87).

WALlersteIn, I. (1984), El moderno sistema mundial, t. 1 et 2 , Madrid, Siglo XXI.

Waldenfels, H. (1997), Manuel de théologie fondamentale, Paris, Cerf. (1988), El capitalismo histórico, Madrid, Siglo XXI.

\section{Résumé}

Afin d'aborder l'altermondialisation et la théologie au Brésil, l'auteur concentre sa réflexion sur trois points. En premier lieu, «Théologie et Église au Brésil » apporte quelques éléments de l'itinéraire d'une Église libératrice dont la place a changé au sein de la société, ce qui, en conséquence, a changé le lieu et la fonction propre de la théologie elle-même. En second lieu, "Théologie et altermondialisation » explicite les bases d'une théologie brésilienne qui a contribué à créer un pont entre la foi chrétienne et la société, selon un principe d' "insertion par contraste» en celle-ci, afin de faire de la 
foi chrétienne et de sa théologie elle-même une prophétie. Enfin, dans "Altermondialisation et théologie ", l'auteur présente, dans leurs grandes lignes, les éléments qui caractérisent l'altermondialisation dans la réflexion théologique au Brésil.

\section{Abstract}

This paper addresses the question of altermondialism and theology in Brazil: it focuses on three points. First, "Theology and the Church in Brazil» brings forward some elements about the evolution of a liberation Church whose place in society has changed. This has also changed the place and function that are specific to theology itself. Second, "Theology and altermondialism" clarifies the bases of a Brazilian theology that contributed to the creation of a bridge between the Christian faith and society. This is the result of an «insertion by contrast» within society, in order to make a prophecy out of the Christian faith and its theology itself. Third, "altermondialism and theology" presents some elements that characterize altermondialism in theological research in Brazil. 